Karl-Franzens Universität Graz

Technische Universität Graz

Medizinische Universität Graz

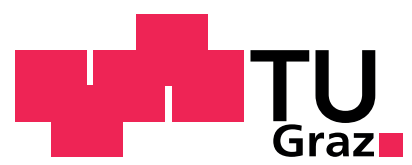

\title{
A Variational Approach to Sparsity Optimization Based on Lagrange Multiplier Theory
}

\author{
K. Ito \\ K. Kunisch
}


SFB sponsors:

- Austrian Science Fund (FWF)

- University of Graz

- Graz University of Technology

- Medical University of Graz

- Government of Styria

- City of Graz

Das Land

Steiermark

Stadt $\mathbf{G} \mathbf{R}$ A Z $\mathbf{Z}$ Wissenschaft 


\title{
A Variational Approach to Sparsity Optimization Based on Lagrange Multiplier Theory
}

\author{
Kazufumi Ito* $\quad$ Karl Kunisch ${ }^{\dagger}$
}

July 6,2012

\begin{abstract}
Sparsity optimization for linear least squares problems formulated as a non-smooth regularization problem is considered in sequence spaces $\ell^{p}$ with $p \in[0,1]$. Necessary optimality conditions in the format of a complementarity system are obtained. A monotonically convergent scheme is developed for the case $p \in(0,1]$. For the case $p=0$ a primal dual active set strategy based on the Lagrange multiplier rule is proposed and analyzed for special cases.
\end{abstract}

Keywords Sparsity optimization, complementarity condition, non-smooth optimization, Lagrange multipliers, primal-dual active set method.

\section{MSC Classification}

\section{Introduction}

In this paper we discuss sparsity optimization for problems of the form:

$$
\min _{x \in \ell^{p}} J(x)=\frac{1}{2}|A x-b|_{2}^{2}+\beta|x|_{p}^{p} .
$$

Here $\ell^{p}=\left\{x \in \ell^{2}: \sum_{k=1}^{\infty}\left|x_{k}\right|^{p}<\infty\right\}, 0<p \leq 1$, is endowed with

$$
|x|_{p}=\left(\sum_{k=1}^{\infty}\left|x_{k}\right|^{p}\right)^{1 / p},
$$

which is a norm if $p=1$ and a quasi-norm for $0<p<1$. We also consider $p=0$. In this case $|x|_{p}^{p}$ in (1.1) is replaced by

$$
|x|_{0}=\text { number of nonzero elements of } x,
$$

and $l^{0}=\left\{x:|x|_{0}<\infty\right\}$. For $x \in l^{0}$ we have that $|x|_{p}^{p} \rightarrow|x|_{0}$, as $p \rightarrow 0^{+}$.

Throughout it is assumed that $A \in \mathcal{L}\left(l^{2}\right)$ and we denote $|x|_{2}=\left(\sum_{k=1}^{\infty}\left|x_{i}\right|^{2}\right)^{1 / 2}$.

Sparsity methods provide an efficient way to extract the essential features of generalized solutions, e.g. in the context of data compression and order reduction methods, with applications arising in signal and image analysis, inverse scattering, de-convolution and tomography problems,

${ }^{*}$ Department of Mathematics, North Carolina State University, Raleigh, North Carolina, 27695-8205, USA; research partially supported by the Army Research Office under DAAD19-02-1-0394

${ }^{\dagger}$ Institut für Mathematik, Karl-Franzens-Universität Graz, A-8010 Graz, Austria, supported in part by the Fonds zur Förderung der wissenschaftlichen Forschung under SFB 32, Mathematical Optimization and Applications in the Biomedical Sciences". 
and wavelet and generalized Fourier analysis. The literature on sparsity optimization is rapidly increasing. Here we mention e.g., [BL, CT, D, WNF, Z] and the references therein. More recently sparsity techniques are also receiving increased attention in the optimal control community, we quote e.g. [CCK, HSW, St] in this respect.

One of the main objectives of this paper is to derive necessary optimality conditions for (1.1) for $0 \leq p \leq 1$ which are of complementarity type. As a consequence, the nature of the conditions is such that they do not require the a-priori knowledge whether a specific coordinate of an optimal solution is different from zero or not. Rather this distinction is built into the optimality condition itself. To obtain this system we us the quadratic nature of the smooth term of the cost. Moreover, since $s \in \mathbb{R} \rightarrow|s|^{p} \in \mathbb{R}^{+}$is not differentiable at $s=0$, problem (1.1) is a non-smooth, nonconvex optimization problem, and hence standard algorithms are not readily available. We develop and analyze algorithms to compute a minimizer for $(1.1)$. In the case $p \in(0,1]$ we use a local regularization of $|x|_{p}^{p}$ and analyze the convergence of a fixed point type scheme. For the case $p=0$ we propose a primal dual active set strategy which is motivated by the optimality condition together with an augmented Lagrangian formulation associated to (1.1).

Let us briefly point to some of the literature that is available for sparsity optimization in the case of $\ell^{p}, p \in[0,1)$ regularization. In [Z] existence for (1.1) is proven and the asymptotic behavior of solution as the regularization parameter tends to zero is analyzed. An iterative algorithm where each step is obtained by solving an optimization subproblem involving a quadratic term with diagonal Hessian plus the original sparsity-inducing regularizer is proposed and analyzed mostly for the

$p=1$ case in $[\mathrm{WNF}]$. In $[\mathrm{BL}]$ a general framework for minimization of non-smooth non-convex functionals based on a generalized gradient projection method is analyzed and applied to (1.1), with $p \in(0,1)$. Iterative hard thresholding techniques were developed in [BD]. In recent papers [LZ, ZDL] penalty decomposition methods are analyzed for for wavelet based image restoration and a general class of nonlinear optimization problems with $\ell^{0}$ regularization terms. The complexity level of the solution to (1.1) as a function of $p$ and $\beta$ is analyzed in [CGWY].

The outline of the paper is as follows. In Section 2 the necessary optimality condition for solutions to (1.1) is derived for $0 \leq p \leq 1$. The complementarity conditions for the cases $p=0$ and $p=1$ are given as well. The asymptotic behavior of the minimizers as $\beta \rightarrow 0^{+}$is analyzed in Section 3. In Section 4 a monotone fixed point algorithm for solving a regularized version of (1.1), $p \in(0,1)$ is analyzed. Section 5 is devoted to an augmented Lagrangian formulation and a primal-dual active set method for the case $p=0$. It is based on the necessary optimality and the Lagrange multiplier approach in Section 2.2. In Section 6 we describe some numerical results obtained for the primal-dual active set method.

\section{Necessary Optimality}

In this section we establish existence and derive necessary optimality conditions for a minimizer of (1.1). For convenience we recall that $l^{r}$ with $1<r<\infty$ are reflexive Banach spaces, that $\left(l^{1}\right)^{\prime}=l^{\infty}$ and $c_{0}^{\prime}=l^{1}$, where $c_{0}$ is the space of all convergent sequences with limit 0 endowed with the sup-norm. We also have $c^{\prime}=l^{1}$, where $c$ is the space of all convergent sequences endowed with the sup-norm, [Y], pg. 115. Moreover for $1 \leq r<s \leq \infty$ we have $l^{r} \varsubsetneqq l^{s}$ and $|x|_{l^{s}} \leq|x|_{l^{r}}$ for all $x \in l^{r}$. 


\subsection{Case $0<p \leq 1$}

To establish existence we use a re-parametrization according to $x=\gamma(y), y \in l^{2}$, where

$$
x_{i}=\gamma(y)_{i}=\left|y_{i}\right|^{\frac{2}{p}} \operatorname{sgn}\left(y_{i}\right), \text { for } i=1, \ldots, \infty .
$$

Note that $y_{i}=\left|x_{i}\right|^{\frac{p}{2}-1} x_{i}$, and that $\gamma: l^{2} \rightarrow l^{p}$ is an isomorphism satisfying $|\gamma(y)|_{p}=|y|_{2}^{\frac{2}{p}}$. In fact, $\gamma$ is clearly injective and for every $x \in l^{p}$ the sequence $\left\{y_{i}\right\}=\left\{\left|x_{i}\right|^{\frac{p}{2}} \operatorname{sgn} x_{i}\right\} \in l^{2}$ provides a preimage under $\gamma$. It follows that (1.1) is equivalent to

$$
\min _{y \in l^{2}} J(y)=\frac{1}{2}|A \gamma(y)-b|_{2}^{2}+\beta|y|_{2}^{2} .
$$

The existence proof for (2.1) will utilize the following result [Z].

Lemma 2.1. The mapping $\gamma: l^{2} \rightarrow l^{2}$ is weakly (sequentially) continuous, i.e. $y^{n} \rightarrow \bar{y}$ weakly in $l^{2}$ implies that $\gamma\left(y^{n}\right) \rightarrow \gamma(\bar{y})$ weakly in $l^{2}$.

Proof. Let $r=\frac{2}{p}+1 \in[3, \infty)$ and let $r^{*}$ denote the conjugate exponent given by $r^{*}=\frac{p}{2}+1 \in\left(1, \frac{3}{2}\right]$. Then $\gamma$ is the duality mapping from $l^{r}$ to $l^{r^{*}}$, i.e.

$$
(\gamma(y), y)_{l^{r^{*}}, l^{r}}=|\gamma(y)|_{r^{*}}|y|_{r}, \quad|\gamma(y)|_{r^{*}}=|y|_{r} .
$$

If $y^{n} \rightarrow \bar{y}$ weakly in $l^{2}$, then $y^{n} \rightarrow \bar{y}$ weakly in $l^{r}$. Since the duality mapping $\gamma: l^{r} \rightarrow l^{r^{*}}$ is weakly sequentially continuous, see [C],pg. 73 , we have $\gamma\left(y^{n}\right) \rightarrow \gamma(\bar{y})$ weakly in $l^{r^{*}}$. Using that $r^{*} \leq 2$, this implies that $\gamma\left(y^{n}\right) \rightarrow \gamma(\bar{y})$ weakly in $l^{2}$.

Theorem 2.1. For any $\beta>0$ there exists a solution $\bar{y} \in l^{2}$ to (2.1), and hence a solution $\bar{x}=$ $\gamma(\bar{y}) \in l^{p}$ to $(1.1)$.

Proof. Let $y^{n}$ be a minimizing sequence of (2.1) and set $x^{n}=\gamma\left(y^{n}\right) \in l^{p}$. Then $\left|y^{n}\right|_{2}^{2}=\left|x^{n}\right|_{p}^{p}$ and thus $x^{n} \in l^{2}$. It follows that $\left\{\left(x^{n}, y^{n}\right)\right\}_{n=1}^{\infty}$ is a bounded sequence in $l^{2} \times l^{2}$. Hence there exists a subsequence, denoted by the same symbols, such that $\left\{\left(x^{n}, y^{n}\right)\right\}$ converges weakly to some $(\bar{x}, \bar{y}) \in l^{2} \times l^{2}$. From Lemma 2.1 we have that $\bar{x}=\gamma(\bar{y})$, and by weak lower semi-continuity of norms we find

$$
\frac{1}{2}|A \gamma(\bar{y})-b|_{2}^{2}+\beta|\bar{y}|_{2}^{2}=\inf _{y \in l^{2}} J(y) .
$$

Hence $\bar{y} \in l^{2}$ is a minimizer of $(2.1)$, and $\bar{x}=\gamma(\bar{y})$ minimizes $(1.1)$.

Since $s \rightarrow \gamma(s)$ is continuous differentiable, we have the necessary optimality condition for $\bar{y}$ :

$$
\gamma^{\prime}\left(y_{i}\right)\left(A_{i}, A \gamma(y)-b\right)+\beta y_{i}=0,
$$

where $A_{i}=A e_{i}$ and $e_{i}$ is the sequence with 1 in the $i-t h$ coordinate and 0 otherwise. It does not provide the one for $\bar{x}$. But we have the following necessary optimality condition for $\bar{y}$ or $\bar{x}$.

Theorem 2.2. If $\bar{x}$ is a global minimizer of (1.1), then

$$
\begin{cases}\bar{x}_{i}=0 & \text { if }\left|\left(A_{i}, f_{i}\right)\right|<\mu_{i} \\ \left(A_{i}, A \bar{x}-b\right)+\frac{\beta p \bar{x}_{i}}{\left|\bar{x}_{i}\right|^{2-p}}=0 & \text { if }\left|\left(A_{i}, f_{i}\right)\right|>\mu_{i},\end{cases}
$$

where $f_{i}=b-A \bar{x}+A_{i} \bar{x}_{i}$ and $\mu_{i}=\beta^{\frac{1}{2-p}}(2-p)(2(1-p))^{-\frac{1-p}{2-p}}\left|A_{i}\right|_{2}^{1-\frac{p}{2-p}}$.

$$
\text { If }\left|\left(A_{i}, f_{i}\right)\right|=\mu_{i} \text {, then } \bar{x}_{i}=0 \text { or } \bar{x}_{i}=\left(\frac{2 \beta(1-p)}{\left|A_{i}\right|_{2}^{2}}\right)^{\frac{1}{2-p}} \operatorname{sgn}\left(\left(A_{i}, f_{i}\right)\right) \text {. }
$$


Proof. Suppose at first that $p \in(0,1)$. The case $p=1$ will be considered below. If $\bar{x}$ is a global minimizer of (1.1), then $\bar{x}_{i} \in \mathbb{R}$ minimizes

$$
F\left(x_{i}\right)=\frac{1}{2}\left|A_{i} x_{i}-f_{i}\right|_{2}^{2}+\beta\left|x_{i}\right|^{p}=\frac{1}{2}\left|A_{i}\right|_{2}^{2} x_{i}^{2}-\left(A_{i}, f_{i}\right) x_{i}+\frac{1}{2}\left|f_{i}\right|_{2}^{2}+\beta\left|x_{i}\right|^{p},
$$

where

$$
f_{i}=b-A \tilde{x}, \quad \tilde{x}_{k}= \begin{cases}0 & k=i \\ \bar{x}_{k} & k \neq i .\end{cases}
$$

Inspection of $F\left(x_{i}\right)$ shows that 0 is a local minimizer of $F\left(x_{i}\right)$. It is the only minimizer if $A_{i}=0$. Henceforth we assume that $A_{i} \neq 0$. If $x_{i}=z>0$ is another local minimizer of (2.3), then

$$
\left|A_{i}\right|_{2}^{2} z-\left(A_{i}, f_{i}\right)+\frac{\beta p}{z^{1-p}}=0
$$

Equation (2.4) has a solution provided that

$$
\left(A_{i}, f_{i}\right) \geq\left|A_{i}\right|_{2}^{\frac{2(1-p)}{2-p}}(p \beta)^{\frac{1}{2-p}}(1-p)^{\frac{p-1}{2-p}}(2-p) .
$$

This follows by requiring that $F^{\prime}(\xi) \leq 0$ where $F^{\prime \prime}(\xi)=0$, i.e. $\xi=\left|A_{i}\right|_{2}^{\frac{2}{p-2}}(\beta p)^{\frac{1}{2-p}}(1-p)^{\frac{1}{2-p}}$. If $F^{\prime}(\xi)<0$, i.e. if the inequality in $(2.5)$ is strict, then $(2.4)$ has two solutions, the smaller one corresponding the a local maximum, the larger to a local minimum of $F\left(x_{i}\right)$. In this case then $F\left(x_{i}\right)$ has two local minima, 0 and $z>0$. To decide whether 0 or $z$ is the global minimizer, we first analyze under which condition $F(z)=F(0)$, i.e.,

$$
\frac{1}{2}\left|A_{i}\right|_{2}^{2}|z|^{2}-\left(A_{i}, f_{i}\right) z+\beta z^{p}=0 .
$$

Then (2.3) has the two distinct global minima. Note that (2.4)-(2.6) are equivalent to

$$
\left\{\begin{array}{l}
\left|A_{i}\right|_{2}^{2} z^{2-p}-\left(A_{i}, f_{i}\right) z^{1-p}+\beta p=0 \\
\frac{1}{2}\left|A_{i}\right|_{2}^{2} z^{2-p}-\left(A_{i}, f_{i}\right) z^{1-p}+\beta=0 .
\end{array}\right.
$$

Subtracting these equation we have

$$
\left|A_{i}\right|_{2}^{2} z^{2-p}=2 \beta(1-p)
$$

and thus

$$
\bar{z}=\left(\frac{2 \beta(1-p)}{\left|A_{i}\right|_{2}^{2}}\right)^{\frac{1}{2-p}}
$$

satisfies $F(\bar{z})=F(0)$. Let us set

$$
\mu_{i}=\left|A_{i}\right|_{2}^{2} \bar{z}+\frac{\beta p}{\bar{z}^{1-p}}=\left|A_{i}\right|_{2}^{\frac{2(1-p)}{2-p}} \beta^{\frac{1}{2-p}}(2(1-p))^{\frac{p-1}{2-p}}(2-p) .
$$

Then from (2.4) we have $F(0)=F(\bar{z})$ if $\left(A_{i}, f_{i}\right)=\mu_{i}$. A short computation shows that $\mu_{i}$ is larger than the expression on the right hand side of (2.5). Moreover, still denoting by $z$ the second local solution of $(2.3)$, we have $F(z)<F(0)$ if and only if $z>\bar{z}$, which is the cases if and only if

$$
\left(A_{i}, f_{i}\right)=\left|A_{i}\right|_{2}^{2} z+\frac{\beta p}{z^{1-p}}>\mu_{i}
$$


Similarly, $F(z)>F(0)$ if and only if $z<\bar{z}$, which holds if and only of $\left(A_{i}, f_{i}\right)<\mu_{i}$. Thus, if the global minimizer of $F\left(x_{i}\right)$ is nonnegative, then necessarily $\bar{x}_{i}=0$, if $\left(A_{i}, f_{i}\right)<\mu_{i}$, and $\bar{x}_{i}=z$ satisfying (2.4), if $\left(A_{i}, f_{i}\right)>\mu_{i}$. If $\left|\left(A_{i}, f_{i}\right)\right|=\mu_{i}$, then $\bar{x}_{i}=0$ or $\bar{x}_{i}=\left(\frac{2 \beta(1-p)}{\left|A_{i}\right|_{2}^{2}}\right)^{\frac{1}{2-p}} \operatorname{sgn}\left(\left(A_{i}, f_{i}\right)\right)$. For $p \rightarrow 0^{+}$, we have $\bar{z} \rightarrow \frac{\sqrt{2 \beta}}{\left|A_{i}\right|_{2}}$ and $\mu_{i} \rightarrow \sqrt{2 \beta}\left|A_{i}\right|_{2}$. The case of nonpositive minima can be treated analogously, by noting that $F\left(x_{i}\right)=\tilde{F}\left(-x_{i}\right)$ where $\tilde{F}\left(x_{i}\right)=\frac{1}{2}\left|A_{i}\right|^{2} x_{i}^{2}+\left(A_{i}, f_{i}\right) x_{i}+\frac{1}{2}\left|f_{i}\right|_{2}^{2}+\beta\left|x_{i}\right|^{p}$.

The case $p=1$ can be treated along the same lines, if the following modifications are taken into account: $\xi=\bar{z}=0$. In particular (2.3) has a unique global minimum which is $x_{i}=0$ if $\left|\left(A_{i}, f_{i}\right)\right| \leq \mu_{i}$, and $x_{i} \neq 0$, satisfying the second equation in $(2.2)$ if $\left|\left(A_{i}, f_{i}\right)\right|>\mu_{i}$. Moreover $\mu_{i}=\beta$ for $p=1$.

From Theorem 2.2 it follows that a minimizer to (1.1) is not necessarily unique. Moreover from its proof we obtain the following corollary.

Corollary 2.1. If $\bar{x}_{i} \neq 0$ then $\left|\bar{x}_{i}\right| \geq\left(\frac{2 \beta(1-p)}{\left|A_{i}\right|_{2}^{2}}\right)^{\frac{1}{2-p}}$.

Indeed the second local solution to (2.3) satisfies necessarily $z \geq \bar{z}$ with $\bar{z}$ given in (2.7).

In $[\mathrm{BL}]$ a necessary optimality condition is obtained for nonlinear problems regularized by $|x|_{p}^{p}$. It considers separately the inactive components with $\bar{x}_{i} \neq 0$ and the active ones. Here we exploit the quadratic nature of the fit-to-data term to obtain a necessary optimality condition which is of complementarity type, separating the active components of $\bar{x}$ from the inactive ones by the sign of $\left|\left(A_{i}, f_{i}\right)\right|-\mu_{i}$.

The following result addresses sparsity of the solution $\bar{x}$ of (1.1). The first part is analogous to a result already contained in $[\mathrm{BL}]$. The second part is applicable in the case that $A$ is close to an orthogonal operator.

Proposition 2.1. Let $\bar{x}$ denote a global minimizer of (1.1). Then we have:

(a) $\#\left\{i: \bar{x}_{i} \neq 0\right\} \leq|b|_{2}^{2}\left(\frac{(2 \beta)^{\frac{2}{p}}}{\sup _{i \in \mathbb{N}}\left|A_{i}\right|_{2}^{2}}(1-p)\right)^{\frac{p}{p-2}}$.

(b) If $\left|\left(A_{i}, b\right)\right|<\mu_{i}-\sup _{j \neq i}\left|\left(A_{i}, A_{j}\right)\right| \sum_{j=1}^{\infty}\left|\bar{x}_{j}\right|$, then $\bar{x}_{i}=0$

Proof. To verify (a) note that by Corollary 2.1

$$
\frac{1}{2}|b|_{2}^{2} \geq \frac{1}{2}|A \bar{x}-b|_{2}^{2}+\beta|\bar{x}|_{p}^{p} \geq \#\left\{i: \bar{x}_{i} \neq 0\right\} \beta\left(\frac{2 \beta(1-p)}{\sup _{i \in \mathbb{N}}\left|A_{i}\right|_{2}^{2}}\right)^{\frac{p}{2-p}}
$$

and hence

$$
\#\left\{i: \bar{x}_{i} \neq 0\right\} \leq \frac{|b|_{2}^{2}}{2 \beta}\left(\frac{2 \beta(1-p)}{\sup _{i}\left|A_{i}\right|_{2}^{2}}\right)^{\frac{p}{p-2}} .
$$

Turning to (b), our assumption implies that

$$
\begin{aligned}
\left|\left(A_{i}, f_{i}\right)\right| & =\left|\left(A_{i}, b-A \bar{x}+A_{i} \bar{x}_{i}\right)\right|=\left|\left(A_{i}, b\right)-\sum_{j \neq i}\left(A_{i}, A_{j}\right) \bar{x}_{j}\right| \\
& \leq\left|\left(A_{i}, b\right)\right|+\left|\sum_{j \neq i}\left(A_{i}, A_{j}\right) \bar{x}_{j}\right| \leq\left|\left(A_{i}, b\right)\right|+\sup _{j \neq i}\left|\left(A_{i}, A_{j}\right)\right| \sum_{j=1}^{\infty}\left|\bar{x}_{j}\right|<\mu_{i},
\end{aligned}
$$

and hence by Theorem 2.2 we have $\bar{x}_{i}=0$. 


\section{$2.2 \quad$ Case $p=0$}

In this section we consider the case $p=0$, i.e,

$$
\min \quad \frac{1}{2}|A x-b|_{2}^{2}+\beta|x|_{0},
$$

where $|x|_{0}=$ number of nonzero elements of $x \in \ell^{2}$. We shall assume that $A \in \mathcal{L}\left(l^{2}\right)$ is radially unbounded, i.e.

$$
|A x|_{2} \rightarrow \infty \text { for }|x|_{2} \rightarrow \infty
$$

Theorem 2.3. Assume that (H1) holds. Then problem (2.8) admits a solution $\bar{x}$ and any weakcluster point in $l^{2}$ of solutions $\left\{x^{p}\right\}$ to (1.1) as $p \rightarrow 0^{+}$is a solution to (2.8). Moreover, the necessary optimality is given by

$$
\begin{cases}\bar{x}_{i}=0 & \text { if }\left|\left(A_{i}, f_{i}\right)\right|<\sqrt{2 \beta}\left|A_{i}\right|_{2} \\ \left(A_{i}, A \bar{x}-b\right)=0 & \text { if }\left|\left(A_{i}, f_{i}\right)\right|>\sqrt{2 \beta}\left|A_{i}\right|_{2},\end{cases}
$$

where $f_{i}=b-A \bar{x}+A_{i} \bar{x}_{i}$. For the second case in (2.9), $\left|\left(A_{i}, f_{i}\right)\right|>\sqrt{2 \beta}\left|A_{i}\right|_{2}$ is equivalent to $\left|\bar{x}_{i}\right|>\frac{\sqrt{2 \beta}}{\left|A_{i}\right|_{2}}$. If $\left|\left(A_{i}, f_{i}\right)\right|=\sqrt{2 \beta}\left|A_{i}\right|_{2}$, then $\bar{x}_{i}=0$ or $\bar{x}_{i}=\frac{\sqrt{2 \beta}}{\left|A_{i}\right|_{2}} \operatorname{sgn}\left(\left(A_{i}, f_{i}\right)\right)$.

Proof. Let $\left\{x^{n}\right\}$ be a minimizing sequence of (2.8). Then there exists $L$ such that $\left|x^{n}\right|_{0} \leq L$ for all $n$. Moreover by (H1) the sequence $\left\{x^{n}\right\}$ is bounded in $l^{2}$. Consequently there exists a weakly convergent subsequence, denoted by the same symbols and a weak limit $\bar{x} \in l^{2}$. In particular $\lim _{n \rightarrow \infty} x_{i}^{n}=\bar{x}_{i}$ for each $i \in \mathbb{N}$. Assume that $|\bar{x}|_{0} \geq L+1$, i.e. $\bar{x}$ contains more than $L$ nontrivial entries. Let $\left\{i_{\ell}\right\}$ be their entries in increasing order. Consider $\left\{\bar{x}_{i_{\ell}}\right\}_{\ell=1}^{L+1}$ and let $\epsilon=\left\{\min \left|\bar{x}_{i_{\ell}}\right|\right.$ : $\ell=1, \ldots, L+1\}$, which satisfies $\epsilon>0$. Since $x_{i_{\ell}}^{n} \rightarrow \bar{x}_{i_{\ell}}$ for $\ell=1, \ldots, L+1$, there exists $n=n(\epsilon)$ such that $x_{i_{\ell}}^{n} \neq 0$ for all $n \geq n(\epsilon)$. This is a contradiction. Hence $i_{L}$ is the largest index for which $\bar{x}_{i} \neq 0$, and hence $|\bar{x}|_{0} \leq L$.

Let $l_{0}^{2}=\left\{x \in l^{2}:|x|_{0}<\infty\right\}$. By the above arguments $\left\{x^{n}\right\} \subset l_{0}^{2}$ and $\bar{x} \in l_{0}^{2}$. We set

$$
\hat{x}_{i}^{n}= \begin{cases}x_{i}^{n} & \text { if } i \leq i_{L} \\ 0 & \text { if } i>i_{L} .\end{cases}
$$

Then

$$
\frac{1}{2}\left|A x^{n}-b\right|_{2}^{2}+\beta\left|\hat{x}^{n}\right|_{0} \leq \frac{1}{2}|A x-b|_{2}^{2}+\beta|x|_{0}, \text { for all } x \in l^{2},
$$

and upon taking liminf with respect to $n$ we obtain that

$$
\min \frac{1}{2}|A \bar{x}-b|_{2}^{2}+\beta|\bar{x}|_{0} \leq \min \frac{1}{2}|A x-b|_{2}^{2}+\beta|x|_{0}, \text { for all } x \in l^{2},
$$

and hence $\bar{x}$ is a solution to (2.8). Next, let $\left\{x^{p}\right\}$ be a solution sequence for (1.1) with $p>0$, as $p \rightarrow 0^{+}$. Thus we have

$$
\frac{1}{2}\left|A x^{p}-b\right|_{2}^{2}+\beta\left|x^{p}\right|_{p}^{p} \leq \frac{1}{2}|A x-b|_{2}^{2}+\beta|x|_{p}^{p} \text { for all } x \in \ell_{0} .
$$

By (H1) the sequence $\left\{x^{p}\right\}$ is bounded in $l^{2}$. Hence there exists a subsequence, denoted by the same symbols that converges weakly in $l^{2}$ to some $\bar{x}$ in $l^{2}$. It satisfies that $x_{i}^{p} \rightarrow \bar{x}_{i}$ for each $i=1, \ldots$, . For $\epsilon>0$ and $x \in l^{2}$ let

$$
N_{\epsilon}(x)_{i}= \begin{cases}1 & \text { if }\left|x_{i}\right|>\epsilon \\ 0 & \text { if }\left|x_{i}\right| \leq \epsilon\end{cases}
$$


Then

$$
\frac{1}{2}\left|A x^{p}-b\right|_{2}^{2}+\beta\left|N_{\epsilon}\left(x^{p}\right) \odot x^{p}\right|_{p}^{p} \leq \frac{1}{2}|A x-b|_{2}^{2}+\beta|x|_{p}^{p} \text { for all } x \in \ell_{0},
$$

where $(a \odot b)_{i}=a_{i} b_{i}$. Using the fact that $\left|N_{\epsilon}(\bar{x})\right|_{0}<\infty$ we find that

$$
\frac{1}{2}|A \bar{x}-b|_{2}^{2}+\beta\left|N_{\epsilon}(\bar{x}) \odot \bar{x}\right|_{0} \leq \frac{1}{2}|A x-b|_{2}^{2}+\beta|x|_{p}^{p} \text { for all } x \in \ell_{0}
$$

Since $\epsilon>0$ was arbitrary this implies that

$$
\frac{1}{2}|A \bar{x}-b|_{2}^{2}+\beta|\bar{x}|_{0} \leq \frac{1}{2}|A x-b|_{2}^{2}+\beta|x|_{p}^{p} \text { for all } x \in \ell_{0},
$$

and hence $\bar{x}$ is a solution to (2.8).

The necessary optimality condition follows from similar arguments as in the proof of Theorem 2.2 .

Condition (H1) on the radial unboundedness of $A$ was used to guarantee existence for (2.8). An alternative to treat (2.8) would be to use a regularization technique by adding $\alpha|x|_{2}^{2}$, for example. - Yet another possibility to guarantee existence consists in requiring that $A$ is radially unbounded from $l^{\infty}$ to $l^{2}$ and that $A \in \mathcal{L}\left(l^{\infty}, l^{2}\right)$ can be expressed as the adjoint of an operator $B \in \mathcal{L}\left(l^{2}, l^{1}\right)$. Then the result remains correct with solution $\bar{x} \in l^{\infty}$. In fact, from a minimizing sequence a subsequence can be extracted with the property that $x^{n} \rightarrow \bar{x}$ weakly* in $l^{\infty}$ and $A x^{n} \rightarrow \bar{y}$ weakly in $l^{2}$. This implies again that $\lim _{n \rightarrow \infty} x_{i}^{n}=\bar{x}_{i}$ for each $i$. Moreover $\left(A x^{n}-A \bar{x}, v\right)_{l^{2}, l^{2}}=\left(x^{n}-\right.$ $\bar{x}, B v)_{l^{\infty}, l^{1}} \rightarrow 0$ for each $v \in l^{2}$, and hence $\lim _{n \rightarrow \infty} A x^{n}=A \bar{x}=\bar{y}$ in $l^{2}$. The remaining parts of the proof can be adjusted accordingly.

We turn to the discussion of sparsity of the solutions to (2.8). To guarantee the necessary a-priori bounds we again utilize (H1). From (2.10) with $x=0$ we have

$$
\frac{1}{2}|A \bar{x}-b|_{2}^{2}+\beta|\bar{x}|_{0} \leq \frac{1}{2}|b|_{2}^{2}
$$

and hence by (H1) the family of solutions $\bar{x}=\bar{x}(\beta)$ to $(2.8)$ is bounded in $\ell^{2}$, i.e. there exists a constant $M$ such that

$$
|\bar{x}(\beta)|_{2} \leq M \text { for all } \beta \in(0, \infty) .
$$

For any $i \in \mathbb{N}$ we introduce

$$
\alpha_{i}=\left(\sum_{j \neq i}\left|\left(A_{i}, A_{j}\right)\right|^{2}\right)^{\frac{1}{2}} \in[0, \infty],
$$

and note that $\alpha_{i}=0$ for all $i$, if $A$ is orthogonal.

Proposition 2.2. Suppose that (H1) holds and let $a^{2}=\sup _{i \in \mathbb{N}}\left|A_{i}\right|_{2}^{2}$. We have

(a) $\#\left\{i: \bar{x}_{i} \neq 0\right\} \leq \frac{a^{2} M^{2}}{2 \beta}$, and

(b) if $\left|\left(A_{i}, b\right)\right| \leq \sqrt{2 \beta}\left|A_{i}\right|-\alpha_{i} M$, then $\bar{x}_{i}=0$

Proof. By Theorem 2.3 and (2.11) we have

$$
M^{2} \geq \sum\left|\bar{x}_{i}\right|_{2}^{2} \geq \sum_{i \in \mathcal{I}} \frac{2 \beta}{\left|A_{i}\right|_{2}^{2}},
$$


where $\mathcal{I}=\left\{i: \bar{x}_{i} \neq 0\right\}$. Consequently

$$
M^{2} \geq \frac{2 \beta}{a^{2}} \#(\mathcal{I})
$$

and (a) follows. Next we compute

$$
\left|\left(A_{i}, f_{i}\right) \leq\right|\left(A_{i}, b\right)|+| \sum_{j \neq i}\left(A_{i}, A_{j}\right) \bar{x}_{j}|\leq|\left(A_{i}, b\right)\left|+\left(\sum_{j \neq i}\left(A_{i}, A_{j}\right)^{2}\right)^{1 / 2} M=\right|\left(A_{i}, b\right) \mid+\alpha_{i} M,
$$

and hence the first case in $(2.12)$ occurs, if $\left|\left(A_{i}, b\right)\right| \leq \sqrt{2 \beta}\left|A_{i}\right|_{2}-\alpha_{i} M$.

Remark 2.1. For those solutions $\bar{x}$ of (2.8) which can be approximated as weak cluster points of solutions $\left\{x^{p}\right\}$, to $(1.1)$ as $p \rightarrow 0^{+}$, we can utilize Proposition 2.1 to establish that $\#\left\{i: \bar{x}_{i} \neq 0\right\} \leq$ $\frac{|b|_{2}^{2}}{2 \beta}$, which has the same asymptotic as Proposition 2.2 (a).

\subsection{Complementarity Problem for $p=0$ and $p=1$}

For $p=1$ the necessary condition of Theorem 2.2 is equivalent to

$$
\begin{gathered}
A^{*}(A x-b)+\beta \lambda=0, \\
\begin{cases}\lambda_{i}=1 & x_{i}>0 \\
\lambda_{i} \in[-1,1] & x_{i}=0 \\
\lambda_{i}=-1 & x_{i}<0 .\end{cases}
\end{gathered}
$$

In fact, if (2.12)-(2.13) hold, then

$$
-\left(A_{i}, f_{i}\right)+\left|A_{i}\right|^{2} x_{i}+\beta \lambda_{i}=0 .
$$

If $\lambda_{i}=1, x_{i}>0$, then $\left|A_{i}\right|_{2}^{2} x_{i}=\left(A_{i}, f_{i}\right)-\beta=\left(A_{i}, f_{i}\right)-\mu_{i}>0$, and if $\lambda_{i}=-1, x_{i}<0$, then $\left|A_{i}\right|_{2}^{2} x_{i}=\left(A_{i}, f_{i}\right)+\beta=\left(A_{i}, f_{i}\right)+\mu_{i}<0$. Consequently $\left(A_{i}, A \bar{x}-b\right)+\beta \frac{x_{i}}{\left|x_{i}\right|}=0$ if $\left|\left(A_{i}, f_{i}\right)\right|>\mu_{i}$, which is the second line of $(2.2)$ for $p=1$. If $x_{i}=0, \lambda_{i} \in[-1,1]$, then $\left(A_{i}, f_{i}\right)-\beta \lambda_{i}=0$ and consequently $\left|\left(A_{i}, f_{i}\right)\right| \leq \mu_{i}$. This is consistent with the first line in (2.2) and the statement concerning the case $\left|\left(A_{i}, f_{i}\right)\right|=\mu_{i}$. Conversely the necessary condition of Theorem 2.2 implies (2.12)-(2.13).

Furthermore, the complementarity condition (2.13) can be expressed as

$$
\lambda_{i}=\frac{\lambda_{i}+c x_{i}}{\max \left(1,\left|\lambda_{i}+c x_{i}\right|\right)}, \text { for all } i=1, \ldots,
$$

for each $c>0$ [IK]. In fact, if (2.14) holds then $\left|\lambda_{i}\right| \leq 1$. If $\left|\lambda_{i}+c x_{i}\right| \leq 1$ then $\lambda_{i}=\lambda_{i}+c x_{i}$ and thus $x_{i}=0$. If $a=\left|\lambda_{i}+c x_{i}\right|>1$, then $\lambda_{i}(a-1)=c x_{i},\left|\lambda_{i}\right|=1$ and thus $\lambda_{i}=\operatorname{sign}\left(x_{i}\right)$. The converse can be argued analogously.

For $p=0$ we introduce the multiplier

$$
\lambda_{i}=\left(A_{i}, b-A \bar{x}\right) .
$$

Then $\left|\left(A_{i}, f_{i}\right)\right|$ can be expressed as $\left|\left(A_{i}, f_{i}\right)\right|=\left.\left|\lambda_{i}+\right| A_{i}\right|^{2} \bar{x}_{i} \mid$ and (2.9) becomes

$$
\begin{cases}\bar{x}_{i}=0 & \text { if }\left.\left.\left|\lambda_{i}+\right| A_{i}\right|^{2} \bar{x}_{i}|<\sqrt{2 \beta}| A_{i}\right|_{2} \\ \lambda_{i}=0 & \text { if }\left.\left.\left|\lambda_{i}+\right| A_{i}\right|^{2} \bar{x}_{i}|>\sqrt{2 \beta}| A_{i}\right|_{2},\end{cases}
$$




\section{$3 \quad$ Asymptotic as $\beta \rightarrow 0^{+}$}

In this section we discuss the asymptotics of $x_{\beta}$ as $\beta \rightarrow 0^{+}$. Let $P$ be the orthogonal projection of $\ell_{2}$ onto $N\left(A^{*}\right)$ and set $\tilde{b}=(I-P) b$. Then,

$$
|A x-b|_{2}^{2}=|A x-(I-P) b|_{2}^{2}+|P b|_{2}^{2}=|A x-\tilde{b}|_{2}^{2}+|P b|_{2}^{2} .
$$

Assume $\tilde{b}=(I-P) b \in R(A)$. Consider the minimum norm problem

$$
\min \quad|x|_{0} \quad \text { subject to } A x-\tilde{b}=0 .
$$

Let $x_{\beta}$ be a minimizer of $(2.8)$ over $x \in \ell_{0}$, given $\beta>0$.

Theorem 3.1. Let $\tilde{b} \in R(A)$ and let (H1) hold. Then every weak cluster point in $\ell^{2}$ of solutions $x_{\beta}$ to $(2.8)$ as $\beta \rightarrow 0^{+}$is a minimizer of $(3.1)$.

Proof. Let $\tilde{x}$ satisfy $A \tilde{x}-\tilde{b}=0$. Then,

$$
\frac{1}{2}\left|A x_{\beta}-\tilde{b}\right|_{2}^{2}+\beta\left|x_{\beta}\right|_{0} \leq \frac{1}{2}|A \tilde{x}-\tilde{b}|_{2}^{2}+\beta|\tilde{x}|_{0}=\beta|\tilde{x}|_{0}
$$

and thus $\left|x_{\beta}\right|_{0} \leq|\tilde{x}|_{0}$ and $\lim \left|A x_{\beta}-\tilde{b}\right|_{2}^{2}=0$ as $\beta \rightarrow 0^{+}$.

The proof is now similar to that of the first part of Theorem 2.3. By (H1) the sequence $\left\{x_{\beta}\right\}_{\beta>0}$ is bounded in $l^{2}$ and hence there exists a weak subsequential limit $\bar{x}$ in $l^{2}$. It clearly satisfies $A \bar{x}=\tilde{b}$. Moreover $|\bar{x}|_{0}<\infty$. Let $i_{L}$ denote the largest index such that $\bar{x}_{i} \neq 0$, and define $\left(\hat{x}_{\beta}\right)_{i}=\left(x_{\beta}\right)_{i}$ if $i \leq i_{L}$ and equal to zero otherwise. Then

$$
\frac{1}{2}\left|A x_{\beta}-\tilde{b}\right|_{2}^{2}+\beta\left|\hat{x}_{\beta}\right|_{0} \leq \frac{1}{2}|A x-\tilde{b}|_{2}^{2}+\beta|x|_{0} \text { for all } x \in l^{2} .
$$

Taking the limit $\beta \rightarrow 0^{+}$concludes the proof.

\section{Monotone Convergent Algorithm}

Here we consider the case $p \in(0,1]$. In order to overcome the singularity of $\left(|s|^{p}\right)^{\prime}=\frac{p s}{|s|^{2-p}}$ near $s=0$, we consider for $\epsilon>0$ the regularized problem:

$$
J_{\epsilon}(x)=\frac{1}{2}|A x-b|_{2}^{2}+\beta \Psi_{\epsilon}\left(|x|^{2}\right),
$$

where for $t \geq 0$

$$
\Psi_{\epsilon}(t)= \begin{cases}\frac{p}{2} \frac{t}{\epsilon^{2-p}}+\left(1-\frac{p}{2}\right) \epsilon^{p} & \text { for } 0 \leq t \leq \epsilon^{2} \\ t^{\frac{p}{2}} & \text { for } t \geq \epsilon^{2},\end{cases}
$$

and $\Psi_{\epsilon}\left(|x|^{2}\right)$ is short for $\sum_{i=1}^{\infty} \Psi_{\epsilon}\left(\left|x_{i}\right|^{2}\right)$. Note that

$$
\Psi_{\epsilon}^{\prime}(t)=\frac{p}{2 \max \left(\epsilon^{2-p}, t^{\frac{2-p}{2}}\right)} \text { for } t \geq 0,
$$

and hence $\Psi \in C^{1}([0, \infty), \mathbb{R})$. The necessary optimality condition for (4.1) is given by

$$
A^{*} A x+\frac{\beta p}{\max \left(\epsilon^{2-p},|x|^{2-p}\right)} x=A^{*} b
$$


where the max-operation is interpreted coordinate-wise. To solve (4.2) we consider the iteration procedure:

$$
A^{*} A x^{k+1}+\frac{\beta p}{\max \left(\epsilon^{2-p},\left|x^{k}\right|^{2-p}\right)} x^{k+1}=A^{*} b,
$$

where the second addend is short for the vector with components $\frac{\beta p}{\max \left(\epsilon^{2-p},\left|x_{i}^{k}\right|^{2-p}\right)} x_{i}^{k+1}$. Multiplying this by $x^{k+1}-x^{k}$, we obtain

$$
\begin{aligned}
& \left.\frac{1}{2}\left(A^{*} A x^{k+1}, x^{k+1}\right)-\frac{1}{2}\left(A^{*} A x^{k}, x^{k}\right)+\frac{1}{2}\left(A^{*} A\left(x^{k+1}-x^{k}\right), x^{k+1}-x^{k}\right)\right) \\
& \quad+\sum_{i=1}^{\infty} \frac{\beta p}{\max \left(\epsilon^{2-p},\left|x_{i}^{k}\right|^{2-p}\right)} \frac{1}{2}\left(\left|x_{i}^{k+1}\right|^{2}-\left|x_{i}^{k}\right|^{2}+\left|x_{i}^{k+1}-x_{i}^{k}\right|^{2}\right)=\left(A^{*} b, x^{k+1}-x^{k}\right) .
\end{aligned}
$$

Below we use that

$$
\frac{1}{\max \left(\epsilon^{2-p},\left|x_{i}^{k}\right|^{2-p}\right)} \frac{p}{2}\left(\left|x_{i}^{k+1}\right|^{2}-\left|x_{i}^{k}\right|^{2}\right)=\Psi_{\epsilon}^{\prime}\left(\left|x_{i}^{k}\right|^{2}\right)\left(\left|x_{i}^{k+1}\right|^{2}-\left|x_{i}^{k}\right|^{2}\right) .
$$

Since $t \rightarrow \Psi_{\epsilon}(t)$ is concave, we have

$$
\Psi_{\epsilon}\left(\left|x_{i}^{k+1}\right|^{2}\right)-\Psi_{\epsilon}\left(\left|x_{i}^{k}\right|^{2}\right)-\frac{1}{\max \left(\epsilon^{2-p},\left|x_{i}^{k}\right|^{2-p}\right)} \frac{p}{2}\left(\left|x_{i}^{k+1}\right|^{2}-\left|x_{i}^{k}\right|^{2}\right) \leq 0
$$

and thus

$$
\left.J_{\epsilon}\left(x^{k+1}\right)+\frac{1}{2}\left(A^{*} A\left(x^{k+1}-x^{k}\right), x^{k+1}-x^{k}\right)\right)+\sum_{i=1}^{\infty} \frac{\beta p}{\max \left(\epsilon^{2-p},\left|x_{i}^{k}\right|^{2-p}\right)} \frac{1}{2}\left|x_{i}^{k+1}-x_{i}^{k}\right|^{2} \leq J_{\epsilon}\left(x^{k}\right) .
$$

We have the following convergence result:

Theorem 4.1. For $\epsilon>0$ let $\left\{x_{k}\right\}$ be generated by (4.3). Then, $J_{\epsilon}\left(x^{k}\right)$ is strictly monotonically decreasing, unless there exists some $k$ such that $x^{k}=x^{k+1}$ and $x^{k}$ satisfies the necessary optimality condition (4.2). Moreover there exists a subsequence of $x_{k}$ that converges weakly in $l^{2}$ to a solution of $(4.2)$.

Proof. From (4.4) it follows that $\left\{x^{k}\right\}_{k=1}^{\infty}$ is bounded in $l^{2}$ and hence in $l^{\infty}$. Consequently from (4.4) there exists $\kappa>0$ such that

$$
\left.J_{\epsilon}\left(x^{k+1}\right)+\frac{1}{2}\left(A^{*} A\left(x^{k+1}-x^{k}\right), x^{k+1}-x^{k}\right)\right)+\kappa\left|x^{k+1}-x^{k}\right|_{2}^{2} \leq J_{\epsilon}\left(x^{k}\right) .
$$

This implies the first part of the theorem. From (4.5) we conclude that

$$
\sum_{k=0}^{\infty}\left|x^{k+1}-x^{k}\right|_{2}^{2}<\infty
$$

Since $\left\{x^{k}\right\}_{k=1}^{\infty}$ is bounded in $\ell^{2}$ there exists $\bar{x} \in \ell^{2}$ and a subsequence such that $x^{k_{\ell}} \rightarrow \bar{x}$ weakly in $\ell^{2}$. By (4.6) moreover $\lim _{\ell \rightarrow \infty} x_{i}^{k_{\ell+1}}=\lim _{\ell \rightarrow \infty} x_{i}^{k_{\ell}}=\bar{x}_{i}$ for all $i$. Testing (4.3) with $e_{i}, i=1, \ldots$ and passing to the limit with respect to $k$, we find that $\bar{x}$ satisfies (4.2). 


\section{Augmented Lagrangian Formulation and Primal-Dual Active Set Method}

In this section we develop the augmented Lagrangian formulation and the primal-dual active strategy for the sparsity optimization problem (2.8). Let $P$ be a nonnegative self-adjoint operator $P$, satisfying $((A+P) x, x) \geq \gamma|x|_{\ell^{2}}^{2}$ for some $\gamma>0$ independent of $x \in \ell^{2}$. We set

$$
\Lambda_{k}=\left|A_{k}\right|_{2}^{2}+\alpha P_{k k}
$$

and let $\Lambda$ denote the invertible diagonal operator with entries $\Lambda_{k}$. Thus, if $A$ is nearly singular, we use $\alpha>0$ and the regularization functional $\frac{\alpha}{2}(x, P x)$ to regularize (2.8). Consider the associated augmented Lagrangian functional

$$
L(x, v, \lambda)=\frac{1}{2}|A x-b|_{2}^{2}+\frac{\alpha}{2}(P x, x)+\beta \sum_{k}\left|v_{k}\right|^{0}+\sum_{k}\left(\frac{\Lambda_{k}}{2}\left|x_{k}-v_{k}\right|^{2}+\left(\lambda_{k}, x_{k}-v_{k}\right)\right) .
$$

Given $(x, \lambda)$, the Lagrangian $L$ is minimized at

$$
v=\Phi(x, \lambda)= \begin{cases}\frac{\lambda_{k}+\Lambda_{k} x_{k}}{\Lambda_{k}} & \text { if }\left|\lambda_{k}+\Lambda_{k} x_{k}\right|^{2}>2 \Lambda_{k} \beta \\ 0 & \text { otherwise. }\end{cases}
$$

Given $(v, \lambda), L$ is minimized at $x$ that satisfies

$$
A^{*}(A x-b)+\alpha P x+\Lambda(x-v)+\lambda=0 .
$$

where $\Lambda$ is diagonal operator with entries $\Lambda_{k}$. Thus, the augmented Lagrangian method [IK] uses the update:

$$
\begin{aligned}
& A^{*}\left(A x^{n+1}-b\right)+\alpha P x^{n+1}+\Lambda\left(x^{n+1}-v^{n}\right)+\lambda^{n}=0 \\
& v^{n+1}=\Phi\left(x^{n+1}, \lambda^{n}\right) \\
& \lambda^{n+1}=\lambda^{n}+\Lambda\left(x^{n+1}-v^{n+1}\right) .
\end{aligned}
$$

If it converges, i.e. $x^{n}, v^{n} \rightarrow x$ and $\lambda^{n} \rightarrow \lambda$, then

$$
\left\{\begin{aligned}
A^{*}(A x-b)+\alpha P x+\lambda=0 & \\
\lambda_{k}=0, & \text { if }\left|\lambda_{k}+\Lambda_{k} x_{k}\right|^{2}>2 \beta \Lambda_{k}, \\
x_{k}=0, & \text { if }\left|\lambda_{k}+\Lambda_{k} x_{k}\right|^{2} \leq 2 \beta \Lambda_{k} .
\end{aligned}\right.
$$

That is, $(x, \lambda)$ satisfies the necessary optimality condition (2.15) with $A$ replaced by $A+P^{\frac{1}{2}}$.

Let us further observe that in the inactive case $\lambda_{k}=0$ and $\left|x_{k}\right|>\sqrt{\frac{2 \beta}{\Lambda_{k}}}$ and in the active case $x_{k}=0$ and $\left|\lambda_{k}\right| \leq \sqrt{2 \beta \Lambda_{k}}$. Thus on the inactive set only the $\lambda$ component is 0 , the $x$ component is different from 0 , on the active the $x$ component is 0 and the $\lambda$ component may or may not be 0 .

Motivated by the augmented Lagrangian formulation we obtain a primal-dual active-set method as follows.

\section{Primal-Dual Active Method (Sparsity optimization)}


1. Initialize: $\lambda^{0}=0$ and determine $x^{0}$ by $A^{*}\left(A x^{0}-b\right)+\alpha P x^{0}=0$. Set $n=0$

2. Solve for $\left(x^{n+1}, \lambda^{n+1}\right)$;

$$
A^{*}\left(A x^{n+1}-b\right)+\alpha P x^{n+1}+\lambda^{n+1}=0,
$$

where

$$
\begin{aligned}
& \lambda_{k}^{n+1}=0, \quad \text { if } k \in\left\{k:\left|\lambda_{k}^{n}+\Lambda_{k} x_{k}^{n}\right|^{2}>2 \beta \Lambda_{k}\right\} \\
& x_{k}^{n+1}=0, \quad \text { if } k \in\left\{k:\left|\lambda_{k}^{n}+\Lambda_{k} x_{k}^{n}\right|^{2} \leq 2 \beta \Lambda_{k}\right\} .
\end{aligned}
$$

3. Converged, or set $k=k+1$ and return to Step 2 .

Note that if the active set method converges, then the converged pair $(x, \lambda)$ satisfies the necessary optimality (5.2).Due to good numerical experience we shall analyze its convergence. First sufficient conditions for uniqueness of solutions to (5.2) will be given and the following remark is made.

Remark 5.1. Since $\left(x^{n+1}, \lambda^{n+1}\right)=0$ for all $n$, we have

$$
\left(\left(A^{*} A+\alpha P\right) x^{n+1}, x^{n+1}\right) \leq\left(A x^{n+1}, b\right)
$$

and thus $\left\{\left|A x^{n}\right|^{2}+\alpha\left(P x^{n}, x^{n}\right)\right\}_{n=1}^{\infty}$ is bounded. Moreover from (5.3) we obtain

$$
\begin{aligned}
0 & =\left(A^{*}\left(A x^{n+1}-b\right)+P x^{n+1}+\lambda^{n+1}, x^{n+1}-x^{n}\right) \\
& =\frac{1}{2}\left(\left|A x^{n+1}-b\right|^{2}+\alpha\left(x^{n+1}, P x^{n+1}\right)\right)-\frac{1}{2}\left(\left|A x^{n}-b\right|^{2}+\alpha\left(x_{n}, P x^{n}\right)\right) \\
& +\frac{1}{2}\left(\left|A\left(x^{n+1}-x^{n}\right)\right|^{2}+\alpha\left(x^{n+1}-x^{n}, P\left(x^{n+1}-x^{n}\right)\right)-\left(\lambda^{n+1}, x^{n}\right) .\right.
\end{aligned}
$$

The term $\left(\lambda^{n+1}, x^{n}\right)$ relates to the switching between active and inactive set. Its value must be controlled to obtain convergence results.

\section{$5.1 \quad$ Uniqueness}

For any pair $(x, \lambda)$ we define

$$
\mathcal{I}(x, \lambda)=\left\{k:\left|\lambda_{k}+\Lambda_{k} x_{k}\right|^{2}>2 \beta \Lambda_{k}\right\} \text { and } \mathcal{A}(x, \lambda)=\left\{k:\left|\lambda_{k}+\Lambda_{k} x_{k}\right|^{2} \leq 2 \beta \Lambda_{k}\right\},
$$

and we set

$$
Q=A^{*} A+P \text {. }
$$

The following diagonal dominance condition will be used:

$$
\left\|\Lambda^{-\frac{1}{2}}(Q-\Lambda) \Lambda^{-\frac{1}{2}}\right\|_{\infty} \leq \rho \text { for some } \rho \in(0,1) .
$$

Theorem 5.1. (Uniqueness) Assume that (5.5) holds. Then there exists at most one solution to (5.2) satisfying

$$
\inf _{\mathcal{I}(x, \lambda)}\left|\Lambda^{-\frac{1}{2}}(\lambda+\Lambda x)\right| \geq(1+\delta) \sqrt{2 \beta}
$$

provided that $\delta>\frac{2 \rho}{1-\rho}$. An analogous statement holds with (5.6) replaced by $\sup _{\mathcal{A}(x, \lambda)} \mid \Lambda^{-\frac{1}{2}}(\lambda+$ $\Lambda x) \mid \leq(1-\delta) \sqrt{2 \beta}$. 
Above $\min _{\mathcal{I}(x, \lambda)}\left|\Lambda^{-\frac{1}{2}}(\lambda+\Lambda x)\right|$ stands for $\min _{k \in \mathcal{I}(x, \lambda)}\left|\Lambda_{k}^{-\frac{1}{2}}\left(\lambda_{k}+\Lambda x_{k}\right)\right|$.

Proof. Assume that there two pairs $(x, \lambda)$ and $(\hat{x}, \hat{\lambda})$ satisfying $(5.2)$ and (5.6). Then we have

$$
Q(x-\hat{x})+\lambda-\hat{\lambda}=0
$$

and therefore

$$
\Lambda^{\frac{1}{2}} x+\Lambda^{-\frac{1}{2}} \lambda-\left(\Lambda^{\frac{1}{2}} \hat{x}+\Lambda^{-\frac{1}{2}} \hat{\lambda}\right)=\Lambda^{-\frac{1}{2}}(\Lambda-Q) \Lambda^{-\frac{1}{2}} \Lambda^{\frac{1}{2}}(x-\hat{x}) .
$$

First consider the case that $x_{k} \neq 0$ if and only if $\hat{x}_{k} \neq 0$. Then, due to the fact that $x_{k} \neq 0$ implies that $\lambda_{k}=0$, diagonal dominance implies that $x=\hat{x}$ and consequently $\lambda=\hat{\lambda}$. If there exists $j$ such that $\operatorname{sign}\left|x_{j}\right| \neq \operatorname{sign}\left|\hat{x}_{j}\right|$, then without loss of generality we may assume that $x_{j} \neq 0$ and $\hat{x}_{j}=0$.

As mentioned below (5.2) we have

$$
\left|\left(\Lambda^{-\frac{1}{2}} \lambda\right)_{k}\right| \leq \sqrt{2 \beta} \text { if } x_{k}=0, \quad \text { and }\left|\left(\Lambda^{-\frac{1}{2}} \hat{\lambda}\right)_{k}\right| \leq \sqrt{2 \beta} \text { if } \hat{x}_{k}=0 .
$$

By (5.7) we find

$$
\Lambda^{\frac{1}{2}}(x-\hat{x})=\Lambda^{-\frac{1}{2}}(\Lambda-Q) \Lambda^{-\frac{1}{2}} \Lambda^{\frac{1}{2}}(x-\hat{x})-\Lambda^{-\frac{1}{2}}(\lambda-\hat{\lambda}) .
$$

Due to (5.6) and (5.8) we obtain

$$
\left|\Lambda^{\frac{1}{2}}(x-\hat{x})\right|_{\infty} \leq \rho\left|\Lambda^{\frac{1}{2}}(x-\hat{x})\right|_{\infty}+2 \sqrt{2 \beta},
$$

and hence

$$
\left|\Lambda^{\frac{1}{2}}(x-\hat{x})\right|_{\infty} \leq \frac{2 \sqrt{2 \beta}}{1-\rho} .
$$

Again by (5.7) and by (5.9) we have for each $k$ :

$$
\begin{gathered}
\left|\Lambda_{k}^{-\frac{1}{2}}\left(\lambda_{k}-\Lambda_{k} x_{k}\right)\right|-\left|\Lambda_{k}^{-\frac{1}{2}}\left(\hat{\lambda}_{k}-\Lambda_{k} \hat{x}_{k}\right)\right| \leq \mid \Lambda_{k}^{-\frac{1}{2}}\left(\lambda_{k}-\hat{\lambda}_{k}+\Lambda_{k}\left(x_{k}-\hat{x}_{k}\right) \mid\right. \\
\left|\Lambda^{-\frac{1}{2}}(\lambda-\hat{\lambda}+\Lambda(x-\hat{x}))\right|_{\infty} \leq \rho\left|\Lambda^{\frac{1}{2}}(x-\hat{x})\right|_{\infty} \leq \frac{2 \rho \sqrt{2 \beta}}{1-\rho},
\end{gathered}
$$

and consequently for the $j$ chosen above

$$
\left|\Lambda_{j}^{-\frac{1}{2}}\left(\lambda_{j}-\Lambda_{j} x_{j}\right)\right|-\left|\Lambda_{j}^{-\frac{1}{2}}\left(\hat{\lambda}_{j}-\Lambda_{j} \hat{x}_{j}\right)\right| \leq \frac{2 \rho \sqrt{2 \beta}}{1-\rho} .
$$

The strict complementarity assumption (5.6) implies that

$$
(1+\delta) \sqrt{2 \beta}-\sqrt{2 \beta} \leq \frac{2 \rho \sqrt{2 \beta}}{1-\rho},
$$

and hence $\delta \leq \frac{2 \rho}{1-\rho}$ which is a contraction to the assumption $\delta>\frac{2 \rho}{1-\rho}$. - The case $\max _{\mathcal{A}(x, \lambda)} \mid \Lambda^{-\frac{1}{2}}(\lambda+$ $\Lambda x) \mid \leq(1-\delta) \sqrt{2 \beta}$ can be treated analogously. 


\subsection{Convergence: Diagonal dominant case}

Here we give a sufficient condition for the convergence of the primal-dual active set method. We shall utilize a diagonal dominance condition and consider a solution to (5.2) which satisfies a strict complementary condition. As such it is unique according to Theorem 5.1. Recall that by Remark (5.1) there exists $M$ such that the iterates are bounded, i.e. $\left|x^{n}\right|_{\ell^{2}} \leq M$ for all $n$. We set $\tilde{M}=\left\|\Lambda^{\frac{1}{2}}\right\|_{\mathcal{L}\left(\ell^{2}, \ell^{\infty}\right)} M$.

Proposition 5.1. Let $(\bar{x}, \bar{\lambda})$ denote a solution to (5.2) which satisfies the strict complementarity condition

$$
\sup _{\mathcal{A}(\bar{x}, \bar{\lambda})}\left|\Lambda^{-\frac{1}{2}} \bar{\lambda}\right| \leq(1-\delta) \sqrt{2 \beta} \text { and } \inf _{\mathcal{I}(\bar{x}, \bar{\lambda})}\left|\Lambda^{\frac{1}{2}} \bar{x}\right| \geq(1+\delta) \sqrt{2 \beta}
$$

and suppose that (5.6) holds. If $0<\delta<\frac{\rho}{1-\rho}\left(\frac{2 \rho \tilde{M}}{\sqrt{2 \beta}}+1\right)$, then the sets

$$
S^{n}=\left\{k \in \mathcal{I}(\bar{x}, \bar{\lambda}): \lambda_{k}^{n}=0\right\} \quad \text { and } T^{n}=\left\{k \in \mathcal{A}(\bar{x}, \bar{\lambda}): x_{k}^{n}=0\right\}
$$

are monotonically nondecreasing. If $S^{n}=S^{n+1}$ and $T^{n}=T^{n+1}$ for some $n$, then $\left(x^{n}, \lambda^{n}\right)=(\bar{x}, \bar{\lambda})$.

The proof will not make use of the particular initialization of the algorithm. In particular, if (5.2) admits a solution satisfying the strict complementarity assumption (5.10) we have global convergence to this solution.

Proof of Proposition 5.2. For two consecutive iterates we have

$$
Q\left(x^{n}-x^{n-1}\right)+\lambda^{n}-\lambda^{n-1}=0,
$$

and thus

$$
\Lambda^{-\frac{1}{2}}(Q-\Lambda) \Lambda^{-\frac{1}{2}} \Lambda^{\frac{1}{2}}\left(x^{n}-x^{n-1}\right)+\Lambda^{-\frac{1}{2}}\left(\lambda^{n}+\Lambda x^{n}\right)-\Lambda^{-\frac{1}{2}}\left(\lambda^{n-1}+\Lambda x^{n-1}\right)=0 .
$$

If $x_{k}^{n}=0$, then either $\lambda_{k}^{n}=0$, or $\lambda_{k}^{n}=0$, in which case $\left|\lambda^{n-1}+\Lambda x^{n-1}\right|^{2} \leq 2 \beta \Lambda_{k}$ and by (5.11)

$$
\begin{aligned}
& \left|\Lambda^{-\frac{1}{2}} \lambda_{k}^{n}\right| \leq\left|\left[\Lambda^{-\frac{1}{2}}(Q-\Lambda) \Lambda^{-\frac{1}{2}} \Lambda^{\frac{1}{2}}\left(x^{n}-x^{n-1}\right)\right]_{k}\right|+\left|\Lambda_{k}^{-\frac{1}{2}}\left(\lambda_{k}^{n-1}+\Lambda_{k} x_{k}^{n-1}\right)\right| \\
& \quad \leq \rho\left|\Lambda^{\frac{1}{2}}\left(x^{n}-x^{n-1}\right)\right|_{\infty}+\sqrt{2 \beta}=2 \rho \tilde{M}+\sqrt{2 \beta}
\end{aligned}
$$

with $\tilde{M}$ as defined before the statement of the theorem. We also have

$$
\lambda\left(x^{n}-\bar{x}\right)+\lambda^{n}-\bar{\lambda}=(\Lambda-Q)\left(x^{n}-\bar{x}\right),
$$

and hence

$$
\Lambda^{\frac{1}{2}}\left(x^{n}-\bar{x}\right)+\Lambda^{-\frac{1}{2}}\left(\lambda^{n}-\bar{\lambda}\right)=\Lambda^{-\frac{1}{2}}(\Lambda-Q) \Lambda^{-\frac{1}{2}} \Lambda^{\frac{1}{2}}\left(x^{n}-\bar{x}\right)
$$

Considering separately the cases $x_{k}^{n} \neq 0, \bar{x}_{k} \neq 0$, and $x_{k}^{n} \neq 0, \bar{x}_{k}=0$, and $x_{k}^{n}=0, \bar{x}_{k} \neq 0$, by (5.6), we find for all $k$ and any $n, \operatorname{using}(5.12)$ and $\left|\Lambda_{k}^{-\frac{1}{2}} \bar{\lambda}_{k}\right| \leq \sqrt{2 \beta}$, that

$$
\left|\Lambda^{\frac{1}{2}}\left(x_{k}^{n}-\bar{x}_{k}\right)\right| \leq \rho\left|\Lambda^{\frac{1}{2}}\left(x^{n}-\bar{x}\right)\right|_{\infty}+2 \rho \tilde{M}+\sqrt{2 \beta}
$$


As a consequence we have

$$
\left|\Lambda^{\frac{1}{2}}\left(x^{n}-\bar{x}\right)\right|_{\infty} \leq \frac{2 \rho \tilde{M}+\sqrt{2 \beta}}{1-\rho} .
$$

Considering (5.13) on the set $S^{n}=\left\{\lambda_{k}^{n}=\bar{\lambda}_{k}=0\right\}$ we find

$$
\sup _{S^{n}}\left|\Lambda^{\frac{1}{2}}\left(x_{k}^{n}-\bar{x}_{k}\right)\right| \leq \rho\left|\Lambda^{\frac{1}{2}}\left(x^{n}-\bar{x}\right)\right|_{\infty} \leq \frac{\rho}{1-\rho}(2 \rho \tilde{M}+\sqrt{2 \beta}) .
$$

For $k \in S^{n}$ we have $\left|\Lambda^{\frac{1}{2}} \bar{x}_{k}\right| \geq(1+\delta) \sqrt{2 \beta}$ and hence

$$
\begin{aligned}
& \left|\Lambda_{k}^{-\frac{1}{2}} \lambda_{k}^{n}+\Lambda_{k}^{\frac{1}{2}} x_{k}^{n}\right| \geq\left|\Lambda_{k}^{\frac{1}{2}} \bar{x}_{k}\right|-\left|\Lambda_{k}^{\frac{1}{2}}\left(x_{k}^{n}-\bar{x}_{k}\right)\right| \geq(1+\delta) \sqrt{2 \beta}-\frac{\rho}{1-\rho}(2 \rho \tilde{M}+\sqrt{2 \beta}) \\
& \quad=\sqrt{2 \beta}\left(1+\delta-\frac{\rho}{1-\rho}(2 \rho \tilde{M}+\sqrt{2 \beta})\right)>\sqrt{2 \beta}
\end{aligned}
$$

and hence $\lambda_{k}^{n}=0$ and $k \in S^{n+1}$. For $k \in T^{n}$ we have by (5.13) and (5.14)

$$
\left|\Lambda_{k}^{-\frac{1}{2}}\left(\lambda_{k}^{n}-\bar{\lambda}_{k}\right)\right| \leq \rho\left|\Lambda^{\frac{1}{2}}\left(x^{n}-\bar{x}\right)\right|_{\infty} \leq \frac{\rho}{1-\rho}(2 \rho \tilde{M}+\sqrt{2 \beta})
$$

and hence

$$
\begin{aligned}
& \left|\Lambda_{k}^{-\frac{1}{2}} \lambda_{k}^{n}+\Lambda_{k}^{\frac{1}{2}} x_{k}^{n}\right|=\left|\Lambda_{k}^{-\frac{1}{2}} \lambda_{k}^{n}\right| \leq\left|\Lambda_{k}^{-\frac{1}{2}}\left(\lambda_{k}^{n}-\bar{\lambda}_{k}\right)\right|+\left|\Lambda_{k}^{-\frac{1}{2}} \bar{\lambda}_{k}\right| \\
& \quad \leq(1-\delta) \sqrt{2 \beta}+\frac{\rho}{1-\rho}(2 \rho \tilde{M}+\sqrt{2 \beta})=\sqrt{2 \beta}\left(1+\frac{\rho}{1-\rho}(2 \rho \tilde{M}+\sqrt{2 \beta})-\delta\right)<\sqrt{2 \beta}
\end{aligned}
$$

and hence $x_{k}^{n+1}=0$ and $k \in T^{n+1}$.

Assume now that $S^{n}=S^{n+1} \subset \mathcal{I}(\bar{x}, \bar{\lambda})$ and $T^{n}=T^{n+1} \subset \mathcal{A}(\bar{x}, \bar{\lambda})$ and that

$$
S^{n} \cup T^{n} \subsetneq \mathcal{I}(\bar{x}, \bar{\lambda}) \cup \mathcal{A}(\bar{x}, \bar{\lambda}) .
$$

Assume that there exists $k \in \mathcal{A}(\bar{x}, \bar{\lambda}) \backslash T^{n}$. Then

$$
\begin{aligned}
& x_{k}^{n+1} \neq 0, \quad x_{k}^{n} \neq 0, \quad \bar{x}_{k}=0, \\
& \lambda_{k}^{n+1}=0, \quad \lambda_{k}^{n}=0 .
\end{aligned}
$$

The update rule of the algorithm and strict complementarity imply that

$$
\left|\Lambda_{k}^{\frac{1}{2}} x_{k}^{n}\right|>\sqrt{2 \beta} \text { and }\left|\Lambda_{k}^{-\frac{1}{2}} \bar{\lambda}_{k}\right| \leq(1-\delta) \sqrt{2 \beta} .
$$

From (5.13) and (5.14)

$$
\left|\Lambda_{k}^{\frac{1}{2}}\left(x_{k}^{n}-\bar{x}_{k}\right)\right| \leq \frac{\rho}{1-\rho}(2 \rho \tilde{M}+\sqrt{2 \beta})+(1-\delta) \sqrt{2 \beta}
$$

and hence

$$
\sqrt{2 \beta}<\left|\Lambda_{k}^{\frac{1}{2}} x_{k}^{n}\right| \leq \frac{\rho}{1-\rho}(2 \rho \tilde{M}+\sqrt{2 \beta})+(1-\delta) \sqrt{2 \beta} .
$$

This implies that $\delta<\frac{\rho}{1-\rho}\left(\frac{2 \rho \tilde{M}}{\sqrt{2 \beta}}+1\right)$ which is impossible by the choice of $\delta$ and thus $T^{n}=\mathcal{A}(\bar{x}, \bar{\lambda})$. 
Similarly, if there exists $k \in \mathcal{I}(\bar{x}, \bar{\lambda}) \backslash S^{n}$ then

$$
\begin{array}{ll}
\lambda_{k}^{n+1} \neq 0, \quad \lambda_{k}^{n} \neq 0, & \bar{\lambda}_{k}=0, \\
x_{k}^{n+1}=0, & x_{k}^{n}=0, \quad \bar{x}_{k} \neq 0 .
\end{array}
$$

As a consequence

$$
\left|\Lambda_{k}^{-\frac{1}{2}} \lambda_{k}^{n}\right| \leq \sqrt{2 \beta} \text { and }\left|\Lambda_{k}^{\frac{1}{2}} \bar{x}_{k}\right|>(1+\delta) \sqrt{2 \beta}
$$

Again by (5.13) and (5.14)

$$
(1+\delta) \sqrt{2 \beta}<\left|\Lambda_{k}^{\frac{1}{2}}\left(x_{k}^{n}-\bar{x}_{k}\right)\right| \leq \frac{\rho}{1-\rho}(2 \rho \tilde{M}+\sqrt{2 \beta})+\sqrt{2 \beta},
$$

which implies that $\delta<\frac{\rho}{1-\rho}\left(\frac{2 \rho \tilde{M}}{\sqrt{2 \beta}}+1\right)$. This is impossible by the choice of $\delta$ and thus $S^{n}=\mathcal{I}(\bar{x}, \bar{\lambda})$. Once the active set structure is determined the unique solution is determined by (5.3).

In the finite dimensional case, we can use the fact that there are only finitely many combinations of active indices and we obtain the following corollary.

Corollary 5.1. In the finite dimensional case under the assumptions of Proposition 5.1 the algorithm converges in finitely many steps.

\subsection{Convergence: M-operator case}

Again we set $Q=A^{*} A+\alpha$. For $\mathcal{C}$ an arbitrary subset of the index set $\{1, \ldots, \infty\}$, let $P_{\mathcal{C}}$ be the projection of $l^{2}$ onto $l_{\mathcal{C}}^{2}=\left\{x \in l^{2}: x_{i}=0\right.$ if $\left.i \neq \mathcal{C}\right\}$. Further let $\mathcal{C}^{c}$ denote the complement of $\mathcal{C}$ and set

$$
Q_{\mathcal{C}}=P_{\mathcal{C}} Q P_{\mathcal{C}}, \quad Q_{\mathcal{C}^{c} \mathcal{C}}=P_{\mathcal{C}^{c}} Q P_{\mathcal{C}}
$$

We assume that $Q$ is an M-operator, i.e.

$$
\left(e_{i}, Q e_{j}\right)_{l^{2}} \leq 0, \text { for all } i \neq j, \quad\left(e_{i}, Q e_{j}\right)_{l^{2}}>0, \text { for all } i,
$$

and

$$
Q_{\mathcal{C}}: l_{\mathcal{C}}^{2} \rightarrow l_{\mathcal{C}}^{2} \text { is continuously invertible, with } Q_{\mathcal{C}}^{-1} x \geq 0 \text { for } 0 \leq x \in l_{\mathcal{C}}^{2}
$$

Note that by definition of $\Lambda$ we have $(Q-\Lambda)_{i i}=0$ for all $i$. We further require the property

$$
(Q-\Lambda)_{i j} \leq 0 \text { for all } i \neq j,
$$

which is clearly satisfied if $Q$ is an M-operator and $P$ is a diagonal operator.

Proposition 5.2. If $Q$ is an M-operator, (5.16) holds, $A^{*} b \geq 0$ and initialization is carried out with $x^{0}>0, \lambda^{0}=0$, then $x^{n} \geq 0, \lambda^{n} \geq 0$ for all $n$ and

$$
\mathcal{A}^{n}=\left\{k:\left|\lambda_{k}^{n}+\Lambda_{k} x_{k}^{n}\right|^{2} \leq 2 \beta \Lambda_{k}\right\}
$$

is monotonically decreasing. If $\mathcal{A}^{n}=\mathcal{A}^{n+1}$ for some $n \geq 0$, then $\left(x^{n+1}, \lambda^{n+1}\right)$ satisfy the necessary optimality condition.

Corollary 5.2. In the finite dimensional case under the assumptions of Proposition 5.2 the algorithm converges in finitely many steps. 
Proof of Proposition 5.2. Let $\mathcal{I}^{n}$ denote the complement of $\mathcal{A}^{n}$.

(i) Note that $\lambda^{1}=\lambda^{0}=0$ on $\mathcal{I}^{0}$ and $x^{0} \geq x^{1}=0$ on $\mathcal{A}^{0}$. Hence

$$
Q\left(x^{1}-x^{0}\right)=-\left(\lambda^{1}-\lambda^{0}\right)=0 \text { on } \mathcal{I}^{0} .
$$

Since $Q$ is an M-operator and $x^{0} \geq x^{1}$ on $\mathcal{A}^{0}$ it follows that $x^{1} \leq x^{0}$ on $\mathcal{I}^{0}$.

Since

$$
Q x^{1}=A^{*} b \geq 0 \text { on } \mathcal{I}^{0},
$$

splitting $P_{\mathcal{I}^{0}} Q$ according to $Q_{\mathcal{I}^{0}}+Q_{\mathcal{I}^{0}, \mathcal{A}^{0}}$, the M-property of $Q$ implies that $x^{1} \geq 0$. Analogously

$$
Q x^{1}+\lambda^{1}=A^{*} b \geq 0 \text { on } \mathcal{A}^{0}
$$

and the fact that $x^{1}=0$ on $\mathcal{A}^{0}, x^{1} \geq 0$ on $\mathcal{I}^{0}$ and the M-property of $Q$ imply that $\lambda^{1} \geq 0$ on $\mathcal{A}^{0}$ and consequently $\lambda^{1} \geq 0$.

Next observe that by (5.16)

$$
\lambda^{1}+\Lambda x^{1}-\left(\lambda^{0}+\Lambda x^{0}\right)=-(Q-\Lambda)\left(x^{1}-x^{0}\right) \leq 0
$$

and hence $A^{0} \supseteq \mathcal{A}^{1}$ and, taking complements, $\mathcal{I}^{0} \subseteq \mathcal{I}^{1}$.

(ii) Assume now that $x^{n} \geq 0, \lambda^{n} \geq 0$ and let $\mathcal{I}^{n}, \mathcal{A}^{n}$ be determined according to (5.4). Let

$$
\hat{\mathcal{I}}^{n}=\left\{k \in \mathcal{I}^{n}: x_{k}^{n} \neq 0\right\}, \hat{\mathcal{A}}^{n}=\left(\hat{\mathcal{I}}^{n}\right)^{c} .
$$

Then

$$
Q_{\hat{\mathcal{I}}^{n}} P_{\hat{\mathcal{I}}^{n}}\left(x^{n+1}-x^{n}\right)=-Q_{\hat{\mathcal{I}}^{n} \hat{\mathcal{A}}^{n}} P_{\hat{\mathcal{A}}^{n}}\left(x^{n+1}-x^{n}\right)-P_{\hat{\mathcal{I}}^{n}}\left(\lambda^{n+1}-\lambda^{n}\right) \leq 0
$$

where we use that due to complementarity of $\left(x^{n}, \lambda^{n}\right)$ we have $P_{\hat{\mathcal{I}}^{n}} \lambda^{n}=0, P_{\hat{\mathcal{I}}^{n}} \lambda^{n+1}=0$, and $P_{\hat{\mathcal{A}}^{n}} x^{n+1}=0$. By (5.17) therefore $x^{n+1} \leq x^{n}$, on $\hat{\mathcal{I}}^{n}$ and consequently $x^{n+1} \leq x^{n}$. As in (i) above we now argue that $x^{n+1} \geq 0$ and $\lambda^{n+1} \geq 0$. Finally

$$
\lambda^{n+1}+\Lambda x^{n+1}-\left(\lambda^{n}+\Lambda x^{n}\right)=-(Q-\Lambda)\left(x^{n+1}-x^{n}\right) \leq 0
$$

and hence $\mathcal{A}^{n+1} \subseteq \mathcal{A}^{n}$.

(iii) If $\mathcal{A}^{n+1}=\mathcal{A}^{n}$ then for all $i \in \mathcal{A}^{n+1}=\left\{k:\left|\lambda_{k}^{n+1}+\Lambda_{k} x_{k}^{n}\right|^{2} \leq 2 \beta \Lambda_{k}\right\}$ we have $x_{k}^{n+1}=0$ and for $k \in \mathcal{I}^{n+1}=\mathcal{I}^{n}=\left\{k:\left|\lambda_{k}^{n+1}+\Lambda_{k} x_{k}^{n}\right|^{2}>2 \beta \Lambda_{k}\right\}$ we find $\lambda_{k}^{n+1}=0$. Moreover $Q x^{n+1}+\lambda^{n+1}=A^{*} b$ and thus $\left(x^{n+1}, \lambda^{n+1}\right)$ satisfies the first order conditions.

\section{Examples}

In this section we discuss examples that demonstrate the efficiency of the primal-dual active set algorithm for the case $p=0$. 


\subsection{Sparsity in a Control Problem}

We consider the linear control system:

$$
\frac{d}{d t} y(t)=\mathcal{A} y(t)+B u(t), \quad y(0)=0
$$

i.e.

$$
y(T)=\int_{0}^{T} e^{\mathcal{A}(T-s)} B u(s) d s .
$$

The control problem consists in finding the control function $u$ that steers the state $y$ to a neighborhood of the desired state $y_{d}$ at the terminal time $T=1$. We discretize the problem in time by the mid-point rule:

$$
A u=\sum_{k=1}^{m} e^{\mathcal{A}\left(T-t_{k+1 / 2}\right)} B u_{k} \Delta t,
$$

where $u \in \mathbb{R}^{m}$ is a discretized control vector with coordinates $u_{k}$ which represent the control values at the mid-point of the intervals $\left(t_{k}, t_{k+1}\right)$. A uniform step-size $\Delta t=1 / 50,(\mathrm{~m}=50)$, is utilized. The solution of the control problem is based on the sparsity formulation (1.1) where $b$ is the discretized target function chosen as the Gaussian distribution $y_{d}(x)=\exp (-100(x-.7) 2)$ centered at $x=.7$.

The primal-dual active set formulation (5.4) with $p=0$ was tested, where the weight matrix $P$ was chosen as the derivative norm, i.e.,

$$
(u, P u)=\sum_{k=1}^{m-1}\left|\frac{u_{k+1}-u_{k}}{\Delta t}\right|^{2} \Delta t .
$$

Our linear system is (normalized) one dimensional controlled heat equation for $y=y(t, x)$ :

$$
y_{t}=y_{x x}+b_{1}(x) v_{1}(t)+b_{2}(x) v_{2}(t), \quad x \in(0,1)
$$

with homogeneous boundary condition $y(t, 0)=y(t, 1)=0$, where the differential operator is discretized in space by the fourth order finite difference approximation [LI] with $n=49$ interior spatial nodes (meshsize $\Delta x=1 / 50)$. We have the two time dependent controls $u=\left(v_{1}, v_{2}\right)$ with corresponding spatial control distributions $b_{i}$ chosen as step functions:

$$
b_{1}(x)=\chi_{(.3, .4)}, \quad b_{2}(x)=\chi_{(.6, .7)}
$$

Since the second control distribution is well within the support of the desired state $y_{d}$ we expect the authority of this control to be much stronger than that of the first one, which is a distance away from the target. Our tests were conducted by incrementally increasing $\beta$ from $\beta=.001$ to .1 . For the results of the table below we initialized by $u_{0}=\left(A^{*} A+\alpha P\right)^{-1} A^{*} b$ for the smallest $\beta$ value and with the solution of the smaller $\beta$ value for the next larger one. Moreover $\lambda_{0}=0$ for all cases.

The method globally converges to a solution for each $\beta$. If we modify the initialization and also choose $u=\left(A^{*} A+\alpha P\right)^{-1} A^{*} b$ and $\lambda=0$ for $\beta=.03$ and $\beta=.1$ the algorithm requires 31 and 27 iterates respectively, and converged to the same solution. As we expected the sparsity increases much faster on the first control $v_{1}$ and becomes completely $v_{1}=0$. Also, we added $10 \%$ noise to $f$ and tested the method. It converges globally and the number of iterates is actually smaller. 
Table 1:

\begin{tabular}{|c|c|c|c|c|c|c|c|c|c|c|c|}
\hline$\beta$ & .001 & .003 & .005 & .007 & .01 & .03 & .05 & .07 & .1 & .3 & .5 \\
\hline no of iterates & 1 & 3 & 16 & 7 & 4 & 29 & 7 & 4 & 2 & 2 & 2 \\
\hline$|u|_{0}$ & 98 & 95 & 79 & 72 & 68 & 22 & 13 & 9 & 7 & 2 & 1 \\
\hline
\end{tabular}

\subsection{M-matrix Example}

Here we report on computations corresponding to Section 5.3. We consider

$$
\min _{x \in \mathbb{R}^{n^{2}}} \frac{1}{2}|A x-b|_{2}^{2}+\beta|x|_{0}
$$

where $A$ is the forward finite difference gradient

$$
A=\left(\begin{array}{c}
G_{1} \\
G_{2}
\end{array}\right)
$$

with $G_{1} \in \mathbb{R}^{n(n+1) \times n^{2}}, G_{2} \in \mathbb{R}^{n(n+1) \times n^{2}}$ given by

$$
G_{1}=I \otimes D, \quad G_{2}=D \otimes I
$$

Here $I$ the $n \times n$ identity matrix, $\otimes$ denotes the tensor product, and $D \in \mathbb{R}^{(n+1) \times n}$ is given by

$$
\left(\begin{array}{rrrrr}
1 & 0 & 0 & \ldots & 0 \\
-1 & 1 & 0 & \ldots & 0 \\
& & & & \\
0 & \ldots & 0 & -1 & 1 \\
0 & \ldots & 0 & 0 & -1
\end{array}\right)
$$

Then $A^{T} A$ is an $M$ matrix coinciding with the 5 - point star discretization on a uniform mesh on a square of the Laplacian with Dirichlet boundary conditions. Moreover (6.1) can be equivalently expressed as

$$
\min _{x \in \mathbb{R}^{n^{2}}} \frac{1}{2}|A x|_{2}^{2}-(x, f)+\beta|x|_{0}
$$

where $f=A^{T} b$. If $\beta=0$ this is a discretized variational form of an elliptic equation and for $\beta>0$ it gives a sparsity enhancing solution for this elliptic equation.

In Table (2) we present the results of the primal-dual active set method for $n=132$ mesh and $\mathrm{f}$ chosen as discretization of $f=10 x_{1} \sin \left(5 x_{2}\right) \cos \left(7 x_{1}\right)$. The active sets convergence monotonically in spite of the fact that $f$ does not have uniform sign. It stops with two consecutive iterates coinciding. If $\mathrm{n}$ is increased to $n=256$ then for $\beta=.1$ the algorithm requires 90 iterations to reach the solution satisfying $|x|=52543$. 
Table 2:

\begin{tabular}{|c|c|c|c|c|c|c|}
\hline$\beta$ & .00001 & .0001 & .001 & .01 & .1 & 1 \\
\hline no of iterates & 4 & 9 & 16 & 38 & 40 & 18 \\
\hline$|x|_{0}$ & 42 & 158 & 510 & 2542 & 12911 & 16384 \\
\hline
\end{tabular}

\subsection{Sparsity and SVM}

An important use of the sparsity optimization is for the Support Vector Machine (SVM) for the classification. We are given training data $\mathcal{D}$, a set of $n$ points of the form

$$
\mathcal{D}=\left\{\left(x_{i}, d_{i}\right) \mid x_{i} \in \mathbb{R}^{m}, d_{i} \in\{-1,1\}\right\}_{i=1}^{n}
$$

where the $d_{i}$ is either 1 or -1 . We want to find the maximum-margin hyperplane that divides the points having $d_{i}=1$ from those having $d_{i}=-1$. Any hyperplane can be written as the set of points $x$ satisfying

$$
w \cdot x-\gamma=0 .
$$

To this end a linear SVM determines the hyperplane $(w, \gamma)$ by the unconstrained minimization of the form [HIB]:

$$
\min \frac{1}{2}|\max (0, y)|^{2}+\beta\left(|w|_{p}+\frac{1}{2} \gamma^{2}\right)
$$

over $u=(w, \gamma)$, where $y=e-D(A w-\gamma e)$ and $\max (0, y)$ measures the degree of misclassification. $D$ is the $n \times n$ diagonal matrix with diagonal $D_{i i}=d_{i}$. That is, the SVM algorithm classifies data into two categories, $\mathcal{R}^{-}$and $\mathcal{R}^{+}$, geometrically separated by the plane $\{x: x \cdot w-\gamma=0\}$, and clustered around the two planes

$$
\begin{aligned}
& \mathcal{R}^{-}=\left\{x \in \mathbb{R}^{m}: x \cdot w-\gamma \leq-1\right\} \\
& \mathcal{R}^{+}=\left\{x \in \mathbb{R}^{m}: x \cdot w-\gamma \geq+1\right\} .
\end{aligned}
$$

The weight $w$ can largely consist of insignificant coefficients, which one may desire to "weed" out, or effectively remove. We introduce sparsity by using $\ell^{p}$ which weeds out unnecessary weights and selects the responsible data. That is, if $w_{j}=0$, the $j$ th descriptor is not used for the classifier (6.3). We tested the sparsity formulation (6.2) for detecting neural activities by the Braingate technolgy $[\mathrm{B}]$. The goal of the technology is to classify neural activity that correlates to specific imagined movements given data recorded from an electrode array implanted in the primary motor cortex of the human brain. The vector $x_{i} \in R^{m}$ describes the firing rate at time $i$, i.e. the $\mathrm{j}$-th component of $x_{i}$ corresponds to the firing rate measurement at the $\mathrm{j}$-th electrode. Test example we used has $96(\mathrm{~m}=96)$ neural channels data $\mathcal{D}$ that records neural firing rate data in time. Figure 2 shows a heat map for weights $w$ for neural nodes with different $p$ with a fixed $\beta=1 . e-3$. The sparsity optimization formulation provides a method to identify the active neurons that are most responsible for each movement. The smaller $p$ the more sparsity is enhanced. We used the successive linearization method of Section 5 to solve the optimization problem (6.2), given $0<p<1$. The method is terminated within 5-6 interacts after attaining the desirable accuracy of solutions.

\section{References}

[BD] T. Blumensath and M. Davies, Iterative thresholding for sparse approximations, Journal of Fourier Analysis and Applications 14 (5) (2008) 629654. 

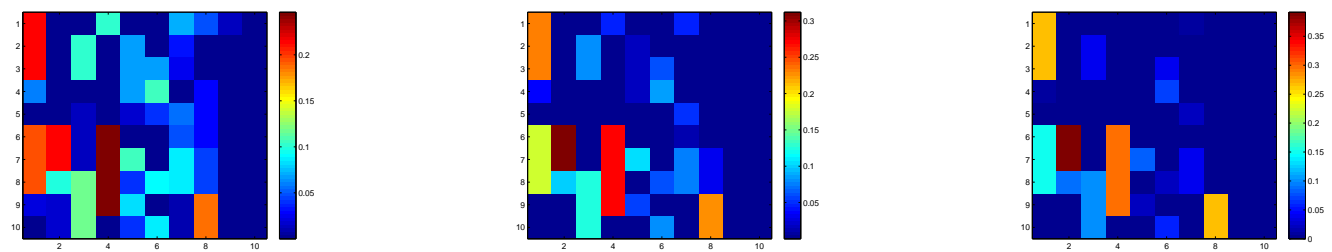

Figure 1: Heat map using $\ell^{1 / 2}, \ell^{1 / 5}$ and $\ell .0002$ norms

[B] C. Bouton: Decoding neural activity from an intracortical implant in humans with tetraplegia. In Biomedical Science \& Engineering Conference, 2009. BSEC 2009. First Annual ORNL, Oak Ridge, TN, March 2009.

[BL] K. Bredies and D.A. Lorenz: Minimization of non-smooth, nonconvex functionals by iterative thresholing, preprint.

[C] I. Cioranescu: Geometry of Banach Spaces, Duality Mappings and Nonlinear Problems, Mathematics and Its Applications, Volume 62, Kluwer Academic Publishers, 1990.

[CCK] E. Casas, C. Clason and K. Kunisch, Approximation of elliptic control problems in measure spaces with sparse solutions, SIAM Journal on Control and Optimization, to appear.

[CT] E. J. Candès and T. Tao: Decoding by linear programming. IEEE Trans. Inform. Theory, 51(12)(2005):4203-4215.

[CGWY] X. Chen, D. Ge, Z. Wang, and Y. Ye, Complexity of Unconstrained L2-Lp Minimization, Math. Programming, to appear.

[D] D. L. Donoho, Compressed Sensing, IEEE Trans.Inform. Theory, 52(2006), 1289-1306.

[HSW] R. Herzog, G. Stadler, and G. Wachsmuth, Directional Sparsity in Optimal Control of Partial Differential Equations, SIAM J. Control Optim., to appear.

[HIB] C. Humber, K. Ito, and C. Bouton: Nonsmooth Formulation of the Support Vector Machine for a Neural Decoding Problem, http://arxiv.org/abs/1012.0958v1.

[IK] K. Ito and K. Kunisch, Lagrange Multiplier Approach to Variational Problems and Applications SIAM Advances in Design and Control, 2008.

[LI] Z. Li and K. Ito, The Immersed Interface Method, SIAM Frontiers in Applied Mathematics, SIAM, Philadelphia, 2006. 
[LJ] R.M. Leahy and B.D. Jeffs On the design of maximally sparse beamforming array IEEE Transaction on Antennas and Propagation, 39(1991), 1178-1187.

[LZ] Z. Lu and Y. Zhang, Sparse approximation via penalty decomposition methods, preprint.

[St] Georg Stadler, Elliptic Optimal Control Problems with $L^{1}$-Control Cost and Applications for the Placement of Control Devices,Comp. Optim. Appls.44(2)(2009),159-181.

[TLY] A.N. Tikhonov, A.S. Leonov, A.G. Yagola, Nonlinear Ill-Posed Problems. Vol. 1, 2, Applied Mathematics and Mathematical Computation, 14, Translated from the Russian, Chapman \& Hall, 1998.

[WNF] S. J. Wright, R. D. Nowak, and M. A. T. Figueiredo, Sparse Reconstruction by Separable Approximation, IEEE Trans. Signal Process, 57 (2009), 2479-2493.

[Y] K. Yosida, Functional Analysis, Springer Verlag, Berlin, 1974.

[Z] C. A. Zarzer. On Tikhonov regularization with non-convex sparsity constraints. Inverse Problems, 25:025006, 2009, 13 pp.

[ZDL] Y. Zhang, B. Dong, and Z. Lu, Minimization for wavelet frame based image restoration, Math. Comp, to appear. 